\title{
Mortalidade neonatal: análise das causas evitáveis
}

\author{
Neonatal mortality: analysis of preventable causes \\ Mortalidad neonatal: análisis de las causas prevenibles
}

\author{
Maria Aparecida Munhoz Gaival ; Elizabeth Fujimori' ; Ana Paula Sayuri Sato ${ }^{I I I}$
}

\begin{abstract}
RESUMO: Trata-se de estudo transversal que teve como objetivo analisar os óbitos neonatais de acordo com a Lista de Causas de Morte Evitáveis por intervenções no âmbito do Sistema Único de Saúde (SUS). Foi desenvolvido em Cuiabá, Mato Grosso com dados do Sistema de Informações sobre Nascidos Vivos e Mortalidade do ano de 2010. Do total de óbitos, 81,1\% eram evitáveis, dos quais $47,3 \%$ por inadequada atenção ao recém-nascido, $21,6 \%$ por inadequada atenção à mulher na gestação e $12,2 \%$ por inadequada atenção ao parto. Conclui-se que o elevado percentual de óbitos neonatais evitáveis por adequada atenção no âmbito do SUS sinaliza que há, no município, condições desfavoráveis de assistência à gestante e ao recém-nascido que reforçam a necessidade de investimentos na estrutura dos serviços e na capacitação dos profissionais.

Palavras-Chave: Mortalidade neonatal; vigilância; sistemas de informação; causa básica de morte.
\end{abstract}

ABSTRACT: This cross-sectional study to examine neonatal deaths in Cuiabá, Mato Grosso against the List of Causes of Death Preventable by Intervention by National Health System (SUS) Services, was conducted with data from the Information Systems on Live Births (SINASC) and Mortality (SIM) for 2010. Of total deaths, $81.1 \%$ were preventable, $47.2 \%$ were due to inadequate care for newborns, $21.6 \%$ to inadequate care for women during pregnancy and $12.2 \%$ to inadequate care during labor. The high percentage of neonatal deaths from inadequate care in the SUS shows unfavorable conditions of antenatal and neonatal care in the municipality, underlining the need for investments in service structure and capacity building for professionals. Keywords: Neonatal mortality; surveillance; information systems; underlying cause of death.

RESUMEN: Se trata de un estudio transversal cuyo objetivo fue analizar las muertes neonatales según a Lista de Causas de muertes prevenibles por intervenciones dentro del Sistema Único de Salud (SUS). Se desarrolló en Cuiabá, Mato Grosso, con datos del Sistema de Información sobre Nacidos Vivos y Mortalidad en el año 2010. Del total de defunciones, un 81.1\% era evitable, entre ellos un $47,2 \%$ se debió a atención inadecuada a los recién nacidos, un $21,6 \%$ a la atención inadecuada a las mujeres durante el embarazo y un $12,2 \%$ a la atención inadecuada durante el parto. Se concluye que el alto porcentaje de las muertes neonatales evitables mediante la atención adecuada en el SUS indica que hay en el municipio, condiciones desfavorables de atención a la embarazada y al recién nacido lo que refuerza la necesidad de inversiones en la estructura de los servicios y en la formación de profesionales.

Palabras Clave: Mortalidad neonatal; vigilancia; sistemas de información; causa básica de muerte.

\section{INTRODUÇÃO}

O coeficiente de mortalidade infantil, que integra os componentes neonatal e pós-neonatal, é um dos melhores indicadores das condições de vida e saúde de uma população ${ }^{1}$.

Nas últimas três décadas, o coeficiente de mortalidade infantil tem diminuído no país, sobretudo pelo decréscimo da mortalidade pós-neonatal (óbitos que ocorrem do $28^{\circ}$ dia até um ano de idade). Contudo, a mortalidade neonatal não tem apresentado mudanças expressivas e, em 2008, foi responsável por $68 \%$ dos óbitos no primeiro ano de vida ${ }^{1}$.
O componente neonatal, que compreende os óbitos ocorridos nos primeiros 27 dias de vida, se distribui em mortalidade neonatal precoce (óbitos que ocorrem nos primeiros 7 dias de vida) e tardia (óbitos ocorridos do $7^{\circ}$ ao $27^{\circ}$ dia de vida), refletindo as condições socioeconômicas e de saúde materna, bem como a qualidade da atenção prestada no pré-natal, parto e ao recém-nascido².

Há evidências de que mais de $70 \%$ dos óbitos neonatais ocorrem por causas evitáveis, especialmente por falta de adequada atenção à gestante e ao recémnascido ${ }^{2-4}$. A prematuridade se destaca como a prin-

\footnotetext{
'Doutora em Enfermagem. Professora Associada 3 da Universidade Federal de Mato Grosso, Faculdade de Enfermagem. Cuiabá, Mato Grosso, Brasil. Pesquisadora do Conselho Nacional de Desenvolvimento Científico e Tecnológico. E-mail: mamgaiva@yahoo.com.br.

IIDoutora em Enfermagem. Professora Associada 3 da Escola de Enfermagem da Universidade de São Paulo, do Departamento de Enfermagem em Saúde Coletiva. São Paulo, Brasil. Pesquisadora do Conselho Nacional de Desenvolvimento Científico. E-mail: efujimor@gmail.com.

IIIDoutora em Enfermagem. Professora Doutora da Faculdade de Saúde Pública da Universidade de São Paulo, do Departamento de Epidemiologia. São Paulo, Brasil.E-mail: sah@usp.br.
} 
cipal causa dos óbitos infantis ocorridos na primeira semana de vida em todas as regiões do país e a segunda causa de óbito é a asfixia/hipóxia nas Regiões Norte e Nordeste, sendo que nas demais regiões predominam as malformações congênitas ${ }^{5}$.

O risco de óbito neonatal é, respectivamente, 44 e 50 vezes maior entre os recém-nascidos com baixo peso $(<2.500 \mathrm{~g})$ e prematuros (idade gestacional $<37$ semanas), da mesma forma que é 54 e 125 vezes maior quando o Apgar é menor que 7 no $1^{\circ}$ e $5^{\circ}$ minuto, respectivamente ${ }^{6}$.

Assim, analisar os óbitos neonatais segundo as causas de morte consideradas evitáveis pode ampliar o entendimento dos acontecimentos patológicos que envolvem o binômio mãe-filho na gestação e no parto e que conduziram à morte ${ }^{2}$. Nesse sentido, vários estudos têm analisado as causas das mortes infantis e neonatais sob o enfoque da evitabilidade ${ }^{3,7-10}$, com vistas a identificar situações que requerem investimentos para a qualificação da atenção à saúde ${ }^{11}$.

Buscando contribuir para a reflexão da temática, o presente estudo teve como objetivo analisar os óbitos neonatais de acordo com a Lista de Causas de Morte Evitáveis por Intervenções no Âmbito do Sistema Único de Saúde (SUS).

\section{ReVisÃo DE LITERATURA}

Define-se como causas evitáveis de óbito, aquelas preveníveis, total ou parcialmente, por ações efetivas dos serviços de saúde que estejam acessíveis em um determinado local e época ${ }^{12}$, delimitando diagnósticos quanto à rede de assistência em saúde.

Muitas causas de óbitos neonatais são consideradas preveníveis a partir de adequado acesso e garantia de assistência de qualidade no que se refere ao pré-natal, parto e puerpério ${ }^{2}$, especialmente por meio de diagnósticos e intervenções precoces e precisos ${ }^{13}$.

Nos últimos anos, a discussão e a produção científica sobre os óbitos infantis evitáveis tem despertado atenção, por assinalar tanto as ações exitosas como as lacunas e fragilidades existentes na organização dos serviços e no processo de trabalho dos profissionais de saúde na atenção à mulher e à criança ${ }^{13}$.

Várias classificações têm sido utilizadas para a análise da evitabilidade do óbito, destacando-se entre elas a Lista de Causas de Mortes Evitáveis por Intervenções no Âmbito do SUS, construída para a realidade brasileira $^{12}$. Para os menores de cinco anos de idade, essa lista classifica: causas evitáveis (reduzíveis por ações de imunoprevenção, por adequada atenção à mulher na gestação, no parto e ao recém-nascido, por ações adequadas de diagnóstico e tratamento e por ações adequadas de promoção à saúde, vinculadas a ações apropriadas de atenção à saúde); causas mal definidas; e demais causas (aquelas não claramente evitáveis).
Dessa forma, identificar e monitorar as causas de óbito no enfoque da evitabilidade representam passos importantes, pois possibilitam a construção de indicadores sensíveis à qualidade da atenção à saúde, acionam mecanismos de investigação para explicação dos óbitos e desencadeiam ações para sua redução ${ }^{14}$.

Portanto, a análise dos óbitos segundo causas potencialmente evitáveis ou reduzíveis por intervenções no âmbito do SUS subsidia a gestão e o planejamento das intervenções preventivas e assistenciais, pois fornece elementos essenciais para o aprimoramento da produção e da atenção em saúde ${ }^{14}$.

\section{Metodologia}

Trata-se de um estudo transversal retrospectivo que analisou dados de mortalidade neonatal ocorridas no ano de 2010 em Cuiabá, capital do Estado de Mato Grosso (MT), que nesse ano contava com uma população de 551.350 habitantes e Índice de Desenvolvimento Humano (IDH) de 0,8215.

Os dados foram obtidos no Sistema de Informação sobre Nascidos Vivos (SINASC) e no Sistema de Informação sobre Mortalidade (SIM) fornecidos pela Gerência de Vigilância de Nascimentos e Óbitos da Secretaria Municipal de Saúde de Cuiabá, MT. Os bancos foram relacionados pela técnica de linkage, com o uso das variáveis: número da declaração de nascimento, nome da mãe e data de nascimento da criança.

No ano de 2010, foram registrados nos sistemas de informação, 9.349 nascidos vivos (NV) e 81 óbitos neonatais. Foram obtidas informações no SINAC referentes a 74 recém-nascidos, o que resultou em vinculação de 91,4\%, com perda de cinco óbitos de recém-nascidos que não constavam nesse sistema e exclusão de dois que eram de mães não residentes em Cuiabá. Assim, foram analisados dados de 74 recémnascidos que faleceram no período neonatal, sendo que 56 foram a óbito no período neonatal precoce e 18 no tardio.

A causa básica de óbito foi obtida no SIM, que tem como referência a Classificação Internacional de Doenças-10a Revisão (CID 10).

Para a análise dos dados, utilizou-se como referencial, a Lista de Causas de Mortes Evitáveis por Intervenções no Âmbito do SUS para menores de cinco anos ${ }^{12}$.

Para o cálculo do coeficiente de mortalidade neonatal $(\mathrm{CMN})$, dividiu-se o número de óbitos de crianças de 0 a 27 dias de vida completos pelo número total de nascidos vivos em 2010 e multiplicou-se o resultado por mil. Procedeu-se da mesma forma para calcular os coeficientes de mortalidade neonatal precoce (CMNP) e tardio (CMNT), quando se utilizou no numerador o número de óbitos de crianças de 0 a 6 dias de vida completos e o número de óbitos de crianças de 7 a 27 dias de vida completos, respectivamente. 
Os bancos de dados originais, arquivados no Tabwin, foram transformados em planilhas do Microsoft Excel 2007 e analisados mediante estatística descritiva, com distribuição de frequências absoluta e relativa.

O projeto de pesquisa foi aprovado pelo Comitê de Ética em Pesquisa (CEP) do Hospital Universitário Júlio Muller (HUJM da Universidade Federal de Nato Grosso), segundo o protocolo no 968/CEPHUJM/2010.

\section{Resultados e Discussão}

O coeficiente de mortalidade neonatal de mães residentes no município de Cuiabá em 2010 foi de 7,9 por mil NV, sendo 6,0 por mil NV no período neonatal precoce, com alta concentração de óbitos nas primeiras 24 horas de vida $(29,7 \%$; $=22)$, e 1,9 por mil NV no período neonatal tardio.

Cerca de um terço das mães era adolescente $(<20$ anos de idade), metade tinha entre 8 e 11 anos de estudo, dois terços não trabalhavam e tiveram menos de sete consultas pré-natais. Maior proporção dos recémnascidos que foram a óbito no período neonatal era do sexo masculino $(54,1 \%)$, de cor não branca $(82,4 \%)$, nascidos pré-termo $(74,3 \%)$ e com baixo peso $(70,3 \%)$, conforme apresentado na Tabela 1.

No ano de 2010, o coeficiente de mortalidade neonatal em Cuiabá (7,9 óbitos por mil NV) ficou abaixo dos coeficientes encontrados em 2009 no país $(10,1$ óbitos por mil NV), no Estado de Mato Grosso (10,3 óbitos por mil NV) e em Cuiabá (10,2 óbitos por mil NV) ${ }^{5,16}$. Entretanto, a despeito dessa diminuição, o índice está longe de ser adequado, pois em países desenvolvidos, como Estados Unidos e Canadá, os coeficientes no ano de 2009 ficaram abaixo de 4 (3,9 óbitos e 2,9 óbitos por mil NV, respectivamente), e mesmo em países da América do Sul, como o Chile (3,8 óbitos por mil NV) ${ }^{17}$. Apesar disso, o coeficiente encontrado em Cuiabá está abaixo do pactuado no Plano Municipal de Saúde 2010-2013, que propôs reduzir a taxa de mortalidade neonatal do município em 5\% ao ano, passando de 10,3 em 2008 para 8,6 óbitos por mil NV até dezembro de $2013^{18}$.

Destacaram-se como causas básicas dos óbitos neonatais, as Afecções originadas no período perinatal $(\mathrm{n}=60)$, sobressaindo-se os Transtornos respiratórios e cardiovasculares específicos do período perinatal, que corresponderam acerca de um terço dos óbitos (35\%), seguidos dos óbitos por Infecção específica do período perinatal (21,6\%). Também se sobressaíram como causas de óbito, os Transtornos relacionados com a duração da gestação e crescimento fetal (13,3\%), especificamente peso muito baixo ao nascer e imaturidade extrema, e Feto e RN afetados por fatores maternos e por complicações da gravidez, trabalho de parto e parto $(13,3 \%)$, como mostra a Tabela 2.
TABELA 1: Distribuição das caracteristicas maternas, da gestação, do parto e dos recém-nascidos que foram a óbito em Cuiabá (MT), Brasil, 2010. ( $N=74)$

\begin{tabular}{|c|c|c|}
\hline Variáveis & $f$ & $\%$ \\
\hline \multicolumn{3}{|l|}{ Maternas } \\
\hline \multicolumn{3}{|l|}{ Idade materna } \\
\hline$<20$ & 23 & 31,1 \\
\hline 20 & 51 & 68,9 \\
\hline \multicolumn{3}{|l|}{ Escolaridade } \\
\hline$<8$ & 19 & 25,7 \\
\hline $8 \mid-12$ & 39 & 52,7 \\
\hline 12 & 16 & 21,6 \\
\hline \multicolumn{3}{|l|}{ Situação conjugal(*) } \\
\hline Com companheiro & 35 & 49,3 \\
\hline Sem companheiro & 36 & 50,7 \\
\hline \multicolumn{3}{|l|}{ Inserção no trabalho } \\
\hline Sim & 25 & 33,8 \\
\hline Não & 49 & 66,2 \\
\hline \multicolumn{3}{|l|}{ Número de filhos vivos } \\
\hline$<2$ & 45 & 60,8 \\
\hline 2 & 29 & 39,2 \\
\hline \multicolumn{3}{|l|}{ Teve filhos mortos } \\
\hline Não & 63 & 85,1 \\
\hline Sim & 11 & 14,9 \\
\hline \multicolumn{3}{|c|}{ Número de consultas de pré-natal(*) } \\
\hline$<7$ & 48 & 66,7 \\
\hline 7 & 24 & 33,3 \\
\hline \multicolumn{3}{|l|}{ Duração da gestação } \\
\hline 37 & 55 & 74,3 \\
\hline$>37$ & 19 & 25,7 \\
\hline \multicolumn{3}{|l|}{ Tipo de parto } \\
\hline Vaginal & 37 & 50 \\
\hline Cesárea & 37 & 50 \\
\hline \multicolumn{3}{|l|}{ Infantis } \\
\hline \multicolumn{3}{|l|}{ Sexo do recém-nascido } \\
\hline Masculino & 40 & 54,1 \\
\hline Feminino & 34 & 45,9 \\
\hline \multicolumn{3}{|c|}{ Raça/cor do recém-nascido } \\
\hline Branca & 13 & 17,6 \\
\hline Não branca & 61 & 82,4 \\
\hline \multicolumn{3}{|l|}{ Peso ao nascer } \\
\hline$<2500$ & 52 & 70,3 \\
\hline 2500 & 22 & 29,7 \\
\hline
\end{tabular}

*Houve informações ignoradas

As Afecções originadas no período perinatal têm sido a principal causa de óbito neonatal no país ${ }^{5,19,20}$. Em Cuiabá, entre essas afecções, os Transtomos respiratórios e cardiovasculares e as Infecções específicas do período perinatal responderam juntas por 35,2\% dos óbitos evitáveis por adequada atenção ao recém-nascido, segundo a Tabela 3. Esse elevado percentual difere do que ocorreu no Brasil de 1997 a 2006, quando os óbitos por causas perinatais como hipóxia intrauterina, asfixia ao nascer e aspiração neonatal diminuíram, provavelmente em decorrência da ampliação do acesso à atenção ao parto e avanços nos cuidados prestados no pós-parto imediato, especialmente pelo acesso aos cuidados neonatais intensivos e uso de surfactante em hospitais do SUS? 
TABELA 2: Distribuição da causa básica do óbito- Cuiabá (MT), Brasil, 2010.

\begin{tabular}{lcc}
\hline \multicolumn{1}{c}{ Causas básicas de óbito (CID X) } & $\mathrm{f}$ & $\%$ \\
\hline Afecções originadas no período perinatal & 60 & 81,1 \\
Transtornos respiratórios e cardiovasculares específicos do período perinatal & 21 & 35,0 \\
Infecções específicas do período perinatal & 13 & 21,6 \\
Transtornos relacionados com a duração da gestação e crescimento fetal & 8 & 13,3 \\
Feto e RN afetados por fatores maternos e por complicações da gravidez, trabalho de parto e parto & 8 & 13,3 \\
Outros transtornos originados no período perinatal & 7 & 11,6 \\
Transtornos endócrinos e metabólicos transitórios e específicos do feto e RN & 2 & 3,3 \\
Traumatismo de parto & 1 & 1,7 \\
Malformações congênitas, deformidades e anomalias cromossômicas & 14 & 18,9 \\
Malformações congênitas do aparelho circulatório & 5 & 6,8 \\
Outras malformações do aparelho digestivo & 3 & 4,1 \\
Malformações congênitas e deformidades aparelho osteomuscular & 2 & 2,7 \\
Outras malformações congênitas & 2 & 2,7 \\
Malformações do sistema nervoso & 2 & 2,7 \\
\hline TOTAL & 74 & 100,0 \\
\hline
\end{tabular}

Análise da mortalidade de neonatos com menos de 32 semanas e $1.500 \mathrm{~g}$ também identificou a síndrome da angústia respiratória como a principal causa de morte e observou que a queda no CMN coincidiu com a implementação de equipamentos e recursos específicos nas unidades neonatais e uso de surfactante e corticoide antenatal ${ }^{21}$.

Em Cuiabá, do total de óbitos analisados, 81,1\% eram evitáveis por intervenções no âmbito do SUS, destacando-se aqueles por adequada atenção ao recém-nascido (47,3\%), à semelhança de resultados obtidos em outros estudos ${ }^{8,20,22}$. Ademais, 21,6\% eram evitáveis por adequada atenção à mulher na gestação e 12,2\% por adequada atenção à mulher no parto, de acordo com a Tabela 3 .

Entre as causas de óbitos evitáveis por adequada atenção ao recém-nascido, destacaram-se os Transtornos respiratórios e cardiovasculares (doença de membrana hialiana e insuficiência respiratória) e a Infecção específica do período perinatal (septicemia). Por adequada atenção à mulher na gestação, sobressaíram como causas de óbito evitáveis, os Transtornos relacionados com a du-

TABELA 3: Distribuição da evitabilidade segundo a causa básica do óbito. Cuiabá (MT), Brasil, 2010.

\begin{tabular}{|c|c|c|}
\hline Classificação de evitabilidade & Causas básicas de óbito (CID X) & $\mathrm{f}$ \\
\hline Causas evitáveis & & 60 \\
\hline \multirow[t]{5}{*}{$\begin{array}{l}\text { Por adequada atenção ao recém- } \\
\text { nascido }\end{array}$} & $\begin{array}{l}\text { Transtornos respiratórios e cardiovasculares específicos do período } \\
\text { perinatal (P22.0, P26.9, P28.0, P28.4, P28.5, P29.3). }\end{array}$ & 13 \\
\hline & Infecção específica do período perinatal (P36.9) & 13 \\
\hline & Outros transtornos originados no período perinatal (P96.8, P96.9). & 7 \\
\hline & $\begin{array}{l}\text { Transtornos endócrinos e metabólicos transitórios e específicos do feto e } \\
\text { RN (P70.0, P70.9). }\end{array}$ & 2 \\
\hline & $\begin{array}{ll}\text { Subtotal } & \text { Sul }\end{array}$ & 35 \\
\hline \multirow[t]{3}{*}{$\begin{array}{l}\text { Por adequada atenção à mulher } \\
\text { na gestação }\end{array}$} & $\begin{array}{l}\text { Transtornos relacionados com a duração da gestação e crescimento fetal } \\
\text { (P07.0, P07.2). }\end{array}$ & 8 \\
\hline & $\begin{array}{l}\text { Feto e RN afetados por fatores maternos e por complicações da gravidez, } \\
\text { trabalho de parto e parto (P00.2, P00.3, P01.1, P01.2, P02.1). }\end{array}$ & 8 \\
\hline & Subtotal & 16 \\
\hline \multirow[t]{3}{*}{ Por adequada atenção ao parto } & $\begin{array}{l}\text { Transtornos respiratórios e cardiovasculares específicos do período } \\
\text { perinatal (P20.9, P21.0, P21.9, P24.0). }\end{array}$ & 8 \\
\hline & Traumatismo de parto (P15.9). & 1 \\
\hline & Subtotal & 9 \\
\hline \multicolumn{2}{|c|}{ Demais causas (não claramente evitáveis) } & 14 \\
\hline & Malformação congênitas do aparelho circulatório (Q21.3, Q22.5, Q24.9) & 5 \\
\hline & Outras malformações do aparelho digestivo (Q39.0, Q39.1, Q43.9) & 3 \\
\hline & $\begin{array}{l}\text { Malformações congênitas e deformidades aparelho osteomuscular } \\
\text { (Q79.0, Q79.3) }\end{array}$ & 2 \\
\hline & Outras malformações congênitas (Q89.7, Q89.9) & 2 \\
\hline & Malformações do sistema nervoso (Q03.1, Q00.0) & 2 \\
\hline Total & & 74 \\
\hline
\end{tabular}


ração da gestação e crescimento fetal (prematuridade e baixo peso ao nascer); e entre os óbitos evitáveis por adequada atenção ao parto, destacaram-se os Transtornos respiratórios e cardiovasculares (asfixia ao nascer e aspiração de mecônio), conforme mostra a Tabela 3 .

Assim, apesar do CMN em Cuiabá ser menor que do Estado e da própria capital no ano anterior, o resultado é preocupante porque mais de $80 \%$ dos óbitos neonatais ocorreram por causas evitáveis. Esse índice corrobora achados de estudo desenvolvido em Recife, também capital de Estado, onde 78,4\% dos óbitos neonatais ocorridos de 1999 a 2009 eram evitáveis ${ }^{3}$. Percentuais elevados de mortes evitáveis também foram observados em uma coorte de crianças de Pelotas, RS, onde 79,2\% dos óbitos ocorridos no primeiro ano de vida eram evitáveis, sendo a maior parte deles $(63,4 \%)$ referente ao óbito neonatal ${ }^{4}$. Outro estudo, também desenvolvido em Pelotas, RS, mostrou que de um total de 266 óbitos infantis ocorridos de 2005 a 2008, 72,9\% eram evitáveis ${ }^{19}$.

Comparado a outro estudo desenvolvido em Cuiabá no ano de $2007^{\circ}$, constatou-se um aumento de quase 10\% nos óbitos evitáveis por adequada atenção ao recém-nascido (de 38\% para 47,3\%) e diminuição de $10,4 \%$ nos óbitos evitáveis por adequada atenção à mulher na gestação, com manutenção dos óbitos que poderiam ser evitados por adequada atenção ao parto.

Esses resultados divergem do que ocorreu no país no período de 1997 a 2006, quando se observou decréscimo de 42,5\% no percentual de óbitos evitáveis por adequada atenção ao recém-nascido, redução de $27,7 \%$ dos óbitos evitáveis por adequada atenção ao parto e aumento de $28,6 \%$ de mortes evitáveis por adequada atenção à mulher na gestação?

Considerando que o acesso à atenção pré-natal tem se ampliado, estranha-se o aumento no percentual de óbitos evitáveis por adequada atenção à mulher na gestação. Estudo que analisou os óbitos ocorridos durante uma década em Recife, PE, verificou que dos 2.267 óbitos evitáveis, 41\% seriam por adequada atenção na gestação ${ }^{3}$. Em Pelotas, RS, dos óbitos infantis ocorridos entre 2004 e 2008, 63,1\% poderiam ter sido evitados por adequada atenção à mulher na gestação ${ }^{4}$, e em Londrina, PR, 32,8\%22. Contudo, da mesma forma que em Cuiabá, em Natal, RN, identificou-se percentual inferior $(22,6 \%)$ de óbitos evitáveis por adequada atenção à mulher na gestação ${ }^{13}$.

O aumento da taxa de cobertura do pré-natal nos últimos anos, em Cuiabá, poderia justificar em parte a diminuição do número de óbitos evitáveis por adequada atenção à mulher na gestação, mas é preocupante o elevado percentual $(66,7 \%)$ de gestantes com menos de 7 consultas pré-natais encontrado entre as mães de recém-nascidos que foram a óbito no período neonatal. Esse resultado é um alerta aos serviços de saúde porque a assistência pré-natal é reconhecida por seu impacto positivo na sobrevida do neonato ${ }^{23,24}$, não se restringindo apenas ao acesso às consultas, mas também no que se refere à qualidade da assistência prestada ${ }^{24,25}$, sendo o prénatal um período propício para a implementação de ações para prevenção e combate à mortalidade neonatal ${ }^{4,25}$.

Embora o baixo peso ao nascer e o nascimento pré-termo resultem de uma complexa rede de determinantes, tais como condições socioambientais e antenatais da saúde materna, a ocorrência desses eventos também está atrelada à qualidade da atenção pré-natal ${ }^{2,6,13}$. Na atualidade, essas são as causas de mortalidade neonatal de maior relevância, e no presente estudo, quase três quartos dos óbitos neonatais (70,3\%) ocorreram em recém-nascidos de baixo peso (inferior a $2.500 \mathrm{~g}$ ), de forma similar ao observado em outra capital de Estado $(74,4 \%)^{3}$. Esse resultado também evidencia a necessidade de se investir na melhoria da qualidade da atenção pré-natal, especialmente porque a prevalência dos nascimentos de baixo peso tem se mantido estável no país desde o início dos anos de 1990, com média de $8 \%$ do total de nascidos vivos ${ }^{26}$.

Dados atuais da Organização Mundial da Saúde classificam o Brasil como o décimo país do mundo com o maior número de nascimentos pré-termo, com prevalência de $9,2 \%{ }^{27}$. Além disso, nos últimos anos constata-se sua ocorrência em mais da metade dos óbitos neonatais, especialmente, nas crianças nascidas com extremo baixo peso ${ }^{28}$.

Quanto aos óbitos evitáveis por adequada atenção à mulher no parto, o percentual encontrado em Cuiabá (12,2\%) assemelha-se aos 13,3\% observados no Brasil em $2006^{7}$ e aos 15,5\% verificados em 16 municípios do Estado de Mato Grosso do Sul, no período de 2000 a $2002^{11}$.

$\bigcirc$ padrão de evitabilidade de óbitos neonatais constatado no presente estudo sugere a existência de problemas relacionados à assistência materno-infantil e indica a necessidade de investimentos na estrutura dos serviços e na capacitação dos profissionais para atender a mulher no ciclo grávido-puerperal e o neonato, prioritariamente no pós-parto imediato.

Conhecer as causas de morte, especialmente aquelas evitáveis por adequada atenção é pois fundamental para avaliar o desempenho dos serviços e monitorar a qualidade da assistência prestada pelo sistema de saúde ${ }^{7,13,14}$.

É importante destacar que, no presente estudo, trabalhou-se com dados de uma capital de Estado brasileiro referente a um ano, de forma que os resultados devem ser interpretados no contexto regional. Entretanto, vale salientar que a análise dos óbitos segundo critérios de evitabilidade representa um importante instrumento para avaliar a qualidade da atenção à saúde. Além disso, a classificação das causas de óbito pode ser considerada como primeiro passo para o melhor conhecimento dos determinantes da mortalidade neonatal na perspectiva de preveni-los ${ }^{14}$. 


\section{ConClusão}

O elevado percentual de óbitos neonatais evitáveis por meio de uma adequada atenção no âmbito do SUS sinaliza que há, no município de Cuiabá, condições desfavoráveis de assistência à gestante e ao recém-nascido que reforçam a necessidade de investimentos na estrutura dos serviços e capacitação dos profissionais, com vistas à melhoria da qualidade da atenção prestada à mulher nos serviços de atendimento pré-natal e ao neonato, especialmente os cuidados prestados no pós-parto imediato.

Uma das limitações do presente estudo refere-se ao fato de se tratar da análise de dados secundários, que depende da fidedignidade e da completude do preenchimento dos registros. $\mathrm{O}$ uso da técnica de linkage de bancos de dados, porém, mostrou-se bastante útil e factível de ser utilizada pelos gestores locais para o enfrentamento da mortalidade neonatal.

Por fim, destaca-se a importância dos sistemas de informação e da análise dos óbitos segundo critérios de evitabilidade, como instrumentos para avaliar e monitorar a qualidade da atenção à saúde.

\section{Agradecimento}

Ao Conselho Nacional de Desenvolvimento Científico e Tecnológico (CNPq). Pesquisa financiada Edital Universal 14/2012, processo no 471609/2012-9.

\section{REFERÊNCIAS}

1.Victora CG, Aquino EML, Leal MC, Monteiro CA, Barros FC, Szwarcwald CL. Maternal and child health in Brazil: progress and challenges. Lancet. 2011; 377:1863-76. 2.Ministério da Saúde (Br). Secretaria de Vigilância em Saúde. Manual de vigilância do óbito infantil e fetal e do Comitê de Prevenção do Óbito Infantil e Fetal. Brasília (DF): Ministério da Saúde; 2009.

3.Rocha R, Oliveira C, Silva DKF, Bonfim C. Mortalidade neonatal e evitabilidade: uma análise do perfil epidemiológico Rev enferm UERJ. 2011; 19:114-20.

4.Gorgot LRMR, Santos I, Valle N, Matisajevich A, Barros AJD, Albernaz E. Óbitos evitáveis até 48 meses de idade entre as crianças da coorte de nascimentos de Pelotas de 2004. Rev Saude Publica. 2011; 45:334-42.

5.Ministério da Saúde (Br). Saúde Brasil 2011: uma análise da situação de saúde e a vigilância da saúde da mulher. Secretaria de Vigilância em Saúde. Brasília (DF): Ministério da Saúde; 2012. [citado em 19 out 2014]. Disponível em: http://portalsaude.saude.gov.br/portalsaude/arquivos/ pdf/2013/Fev/21/saudebrasil2011_parte1_cap1.pdf

6.Maran E, Uchimura TT. Mortalidade neonatal: fatores de risco em um município no sul do Brasil. Revista Eletrônica de Enfermagem. [Internet]. 2008. [citado em 16 out 2014]. Disponível em: http://www.fen.ufg.br/ revista/v10/n1/v10n1a03.htm

7.Malta DC, Duarte EC, Escalante JJC, Almeida MF, Sardinha LMV, Macário EM et al. Mortes evitáveis em menores de um ano, Brasil, 1997 a 2006: contribuições para a avaliação de desempenho do Sistema Único de Saúde. Cad Saúde Pública. 2010; 26:481-91.

8.Jobim R, Aerts D. Mortalidade infantil evitável e fatores associados em Porto Alegre, Rio Grande do Sul, Brasil, 2000-2003. Cad Saúde Pública. 2008; 24:179-87.

9.Lourenço EC, Bruken GS, Luppi CG. Mortalidade infantil neonatal: estudo das causas evitáveis em Cuiabá, Mato Grosso, 2007. Epidemiol Serv Saúde. 2013; 22:697-706. 10.Jodas DA, Scochi MJ, Vicente JB, Colucci AG. Análise dos óbitos evitáveis de menores de cinco anos no município de Maringá-Pr. Esc Anna Nery. 2013; 17:263-70.

11.Gastaud ALGS, Honer MR, Cunha RV. Mortalidade infantil e evitabilidadae em Mato Grosso do Sul, Brasil, 2000 a 2002. Cad Saúde Pública. 2008; 24:1631-40.

12.Malta DC, Duarte EC, Almeida MF, Dias MAS, Moraes Neto OL, Moura et al. Lista de causas de mortes evitáveis por intervenções do Sistema Único de Saúde do Brasil. Epidemiol Serv Saúde. 2007; 16:233-44.

13.Brandão ICA, Godeiro ALS, Monteiro AI. Assistência de enfermagem no pré-natal e evitabilidade de óbitos neonatais. Rev enferm UERJ. 2012; 20:596-602.

14.Malta DC, Duarte EC. Causas de mortes evitáveis por ações efetivas dos serviços de saúde: uma revisão da literatura. Ciênc saúde coletiva. 2007; 12:765-76.

15.Mato Grosso (Br). Secretaria de Estado Planejamento e Coordenação Geral [Internet]. Mato Grosso em números. [citado em 18 out 2014] Disponível em: http://www.seplan.mt.gov.br/sitios/indicador/mtemnumeros2010/pdf/aspectossociais.pdf

16.Cuiabá (MT). Secretaria Municipal de Saúde de Cuiabá. Indicadores de mortalidade de Cuiabá e referencial comparativo - 1997/2009. Cuiabá (MT): Secretaria Municipal de Saúde; 2010.

17.Rajaratnam JK, Marcus JR, Flaxman AD, Wang H, Levin-Rector A, Dwyer L et al. Neonatal, post neonatal, childhood, and under-5 mortality for 187 countries, 1970-2010: a systematic analysis of progress towards Millennium Development Goal 4. The Lancet. [Internet]. 2010. [cited in 2014 Aug 16]. Available in: http:// www.thelancet.com/journals/lancet/article/PISO140. 6736(10)60703-9/abstract

18.Cuiabá (MT). Secretaria Municipal de Saúde de Cuiabá. Plano municipal de saúde 2010-2013. Cuiabá (MT): Secretaria Municipal de Saúde; 2010.

19.Silva VLS, Santos IS, Medronha NS, Matijasecich A. Mortalidade infantil na cidade de Pelotas, Estado do Rio Grande do Sul, Brasil, no período de 2005-2008: uso da investigação de óbitos na análise das causas evitáveis. Epidemiol Serv Saúde. 2012; 21:256-74.

20.Ferrari RAP, Bertolozzi MR, Dalmas JC, Girotto E. Determining factors for neonatal mortality in a city in the Southern Region of Brazil. Rev esc enferm USP. [Internet]. 2013. [cited in 2014 Jun 22]. Available in: http://www.scielo. br/pdf/reeusp/v47n3/en_0080-6234-reeusp-47-3-00531.pdf 21.Barría-Pailaquilén RM, Mendoza-Maldonado Y, Urrutia-Toro Y, Castro-Mora C, Santander-Manríquez G.Trends in infant mortality rate and mortality for neonates born at less than 32 weeks and with very low birth weight. Rev Latino-Am Enfermagem. 2011; 19:977-84. 
22.Santos HG, Andrade SM, Silva AMR, Mathias TAF, Ferrari LL, Mesas AE. Mortes infantis evitáveis por intervenções do Sistema Único de Saúde: comparação de duas cortes de nascimentos. Ciênc saúde coletiva. 2014; 19: 907-16.

23.Kassar SB, Melo ANC, Coutinho SB, Lima MC, Lira PIC. Determinants of neonatal death with emphasis on health care during pregnancy, childbirth and reproductive history. J Pediatr. 2013; 89:269-77.

24. Nascimento RM, Leite AJM Almeida NMGS, Almeida PC, Silva CF. Determinantes da mortalidade neonatal: estudo caso-controle em Fortaleza, Ceará, Brasil. Cad Saúde Pública. 2012; 28:559-72

25.Borba GG, Neves ET, Arrué AM, Silveira A, Zamberlan KC. Fatores associados à morbimortalidade neonatal: um estudo de revisão. Saúde (Santa Maria). 2014; 40:09-16

26.Silva AAM, Silva LM, Barbieri MA, Bettiol H, Carvalho LM, Ribeiro VS, Goldani MZ. The epidemiologic paradoxo flow birth weight in Brazil. Rev Saude Publica. 2010; 44: 767-75.

27.World Health Organization. Born too soon: the global action report on preterm birth. Geneva (Swi): WHO; 2012.

28.Almeida MF, Alencar GP, Schoeps D, Novaes HMD, Campbell O, Rodrigues LC. Sobrevida e fatores de risco para mortalidade neonatal em uma coorte de nascidos vivos de muito baixo peso ao nascer, na Região Sul do Município de São Paulo, Brasil. Cad Saúde Pública. 2011; 27:1088-98. 